International Mathematical Forum, 2, 2007, no. 35, 1717 - 1721

\title{
Round Fuzzy Metric Spaces
}

\author{
Hakan Efe \\ Department of Mathematics \\ Faculty of Science and Arts \\ Gazi University, Teknikokullar \\ 06500 Ankara, Turkey \\ hakanefe@gazi.edu.tr
}

\begin{abstract}
The purpose of this paper to introduce the concept of roundness is fuzzy metric spaces. Also some theorems and definitions is given on round fuzzy metric spaces.
\end{abstract}

Mathematics Subject Classification: 54A40, 54E35

Keywords: Fuzzy metric spaces, round metric spaces, closed sets

\section{Introduction}

In 1965, the concept of fuzzy set was introduced by Zadeh [10]. Many authors have introduced the concept of fuzzy metric space in different ways [1-5]. George and Veeramani [3] modified the concept of fuzzy metric space introduced by Kramosil and Michalek [6] and defined a Hausdorff topology on this fuzzy metric space. Nathanson [7] introduced the concept of round metric space and gave the relationship between round metrics and equivalent metrics.

In this paper we study on the concept of round fuzzy metric space in the sense of George and Veeramani.

\section{Preliminaries}

Definition 2.1[8]: A binary operation $*:[0,1] \times[0,1] \rightarrow[0,1]$ is continuous t-norm if $*$ is satisfying the following conditions:

(i) $*$ is commutative and associative;

(ii) $*$ is continuous;

(iii) $a * 1=a$ for all $a \in[0,1]$;

(iv) $a * b \leq c * d$ whenever $a \leq c$ and $b \leq d$, and $a, b, c, d \in[0,1]$. 
Definition 2.2[3]: A 3-tuple $(X, M, *)$ is said to be a fuzzy metric space if $X$ is an arbitrary set, $*$ is a continuous t-norm and $M$ is a fuzzy set on $X^{2} \times(0, \infty)$ satisfying the following conditions: for all $x, y, z \in X, s, t>0$,

(i) $M(x, y, t)>0$,

(ii) $M(x, y, t)=1$ if and only if $x=y$,

(iii) $M(x, y, t)=M(y, x, t)$,

(iv) $M(x, y, t) * M(y, z, s) \leq M(x, z, t+s)$,

(v) $M(x, y,):.(0, \infty) \rightarrow[0,1]$ is continuous.

Remark 2.3: In fuzzy metric space $X, M(x, y,$.$) is non-decreasing for all$ $x, y \in X$.

Example 2.4: Let $(X, d)$ be a metric space. Denote $a * b=a b$ for all $a, b \in[0,1]$ and let $M_{d}$ be a fuzzy set on $X^{2} \times(0, \infty)$ defined as follows:

$$
M_{d}(x, y, t)=\frac{k t^{n}}{k t^{n}+m d(x, y)}
$$

for all $k, m, n \in \mathbb{R}^{+}$. Then $\left(X, M_{d}, *\right)$ is a fuzzy metric space.

Remark 2.5: Note the above example holds even with the t-norm $a * b=$ $\min \{a, b\}$ and hence $M$ is a fuzzy metric with respect to any continuous t-norm. In the above example by taking $k=m=n=1$, we get

$$
M_{d}(x, y, t)=\frac{t}{t+d(x, y)}
$$

We call this fuzzy metric induced by a metric $d$ the standard fuzzy metric.

Definition 2.6[3]: Let $(X, M, *)$ be a fuzzy metric space and let $r \in(0,1)$, $t>0$ and $x \in X$. The set

$$
B(x, r, t)=\{y \in X: M(x, y, t)>1-r\}
$$

is called the open ball with center $x$ and radius $r$ with respect to $t$.

Theorem 2.7[3]: Every open ball $B(x, r, t)$ is an open set.

Definition 2.8[3]: Let $(X, M, *)$ be a fuzzy metric space and let $r \in(0,1)$, $t>0$ and $x \in X$. The set

$$
B[x, r, t]=\{y \in X: M(x, y, t) \geq 1-r\}
$$

is called the closed ball with center $x$ and radius $r$ with respect to $t$.

Theorem 2.9[3]: Every closed ball $B[x, r, t]$ is a closed set.

Remark 2.10: Let $(X, M, *)$ be a fuzzy metric space. Define $\tau=\{A \subset X$ : for each $x \in X$, there exist $t>0, r \in(0,1)$ such that $B(x, r, t) \subset A\}$. Then $\tau$ is a topology on $X$.

Remark 2.11:

(i) Since $\left\{B\left(x, \frac{1}{n}, \frac{1}{n}\right): n=1,2, \ldots\right\}$ is a local base at $\mathrm{x}$, the topology $\tau$ is first countable.

(ii) Every fuzzy metric space is Hausdorff.

(iii) Let $(X, M, *)$ be an fuzzy metric space and $\tau$ be the topology on $X$ induced by the fuzzy metric. Then for a sequence $\left(x_{n}\right)_{n}$ in $X, x_{n} \rightarrow x$ if and only if $M\left(x_{n}, x, t\right) \rightarrow 1$ as $n \rightarrow \infty$. 
(iv) In a fuzzy metric space every compact set is closed and bounded.

Definition 2.12[9]: Let $(X, M, *)$ be a fuzzy metric space, $x \in X$ and $A \subset X$. The distance between $x$ and $A$ is defined by

$$
M(A, x, t)=\sup \{M(y, x, t): y \in A\}
$$

for all $t>0$.

\section{Main Results}

Let $(X, M, *)$ be a fuzzy metric space. If $A \subset X$, let $\bar{A}$ denote the closure of $A$ in $X$. Then $\overline{B(x, r, t)} \subset B[x, r, t]$ for all $x \in X$, each $r \in(0,1)$ and each $t>0$.

Definition 3.1: Let $(X, M, *)$ be a fuzzy metric space. We call $M$ a round fuzzy metric for $X$ if $\overline{B(x, r, t)}=B[x, r, t]$ for all $x \in X$, each $r \in(0,1)$ and each $t>0$.

Remark 3.2: Let $(X, M, *)$ be a fuzzy metric space. The fuzzy metric $M$ is round iff $y \in \overline{B(x, 1-M(x, y, t), t)}$ for all $x, y \in X$ with $x \neq y$ and each $t>0$.

Definition 3.3: A fuzzy metrizable space $X$ is a round fuzzy metric space if there exist a round fuzzy metric for $X$.

Example 3.4: Let $X=\mathbb{R}$. Define $a * b=a b$ and

$$
M(x, y, t)=\frac{t}{t+|x-y|} .
$$

Then $(X, M, *)$ is a round fuzzy metric space.

Theorem 3.5: Let $X=A \cup K$ be a fuzzy metrizable space, where $A$ and $K$ are nonempty, disjoint, closed sets and $K$ is compact. Then no fuzzy metric for $X$ is round.

Proof. Let $M$ be a fuzzy metric for $X$. Choose $a \in A$. Since $K$ is compact, there exists $k \in K$ such that $M(a, K, t)=\sup \{M(a, x, t): x \in K\}=$ $M(a, k, t)$. Then $B(a, 1-M(a, k, t), t) \subset A$. Since $A$ is closed,

$\overline{B(a, 1-M(a, k, t), t)} \subset A$, and so $k \notin \overline{B(a, 1-M(a, k, t), t)}$. Therefore, $M$ is not a round metric for $X$.

Conclusion 3.6: If a fuzzy metric space $(X, M, *)$ has an isoleted point, then no metric for $X$ is round.

Theorem 3.7: Let $\left(X_{1}, M_{1}, *\right)$ and $\left(X_{2}, M_{2}, *\right)$ be two fuzzy metric spaces without isoleted points, and let $f: X_{1} \rightarrow X_{2}$ be a surjection such that for $x, y, z \in X_{1}$, if $M_{1}(x, z, t)>M_{1}(x, y, t)$, then $M_{2}(f(x), f(z), t)>M_{2}(f(x), f(y), t)$ for all $t>0$. If $M_{1}$ is a fuzzy round metric for $X_{1}$, then $M_{2}$ is a fuzzy round metric for $X_{2}$.

Proof. We first show that $f$ is continuous. Let $x \in X_{1}$, and let $\left(y_{n}\right)_{n}$ be a sequence in $X_{1}$ such that $M_{1}\left(y_{n}, x, t\right)<M_{1}\left(y_{n+1}, x, t\right)$ for all $n \geq 1, t>0$ and 
such that $y_{n} \rightarrow x$. Then

$$
M_{2}\left(f\left(y_{n}\right), f(x), t\right)<M_{2}\left(f\left(y_{n+1}\right), f(x), t\right)
$$

for all $n \geq 1$ and for all $t>0$. Let $r=\sup \left\{M_{2}\left(f\left(y_{n}\right), f(x), t\right): t>0\right\}$. Suppose $r<1$. Since $f(x)$ is not an isolated point of $X_{2}$, there exists $f(z) \in$ $B(f(x), 1-r, t)$ with $z \neq x$ for all $t>0$. Then

$$
M_{2}(f(z), f(x), t)>r \geq M_{2}\left(f\left(y_{n}\right), f(x), t\right)
$$

for all $n \geq 1$ and for all $t>0$. But this is impossible, since $\left(M_{1}\left(y_{n}, x, t\right)\right)_{n}$ increases to 1 . Therefore, $r=1$, the sequence $\left(f\left(y_{n}\right)\right)_{n}$ converges to $f(x)$, and the function $f$ is continuous (In fact, $f$ is a homeomorphism of $X_{1}$ onto $X_{2}$ ).

Let $f(x), f(y) \in X_{2}$ with $x \neq y$. If $M_{1}$ is a round fuzzy metric for $X_{1}$, then $y \in \overline{B\left(x, 1-M_{1}(x, y, t), t\right)}$, and so there is a sequence $\left(z_{n}\right)_{n}$ in $X_{1}$ such that $M_{1}\left(z_{n}, x, t\right)>M_{1}(y, x, t)$ and $\left(z_{n}\right)_{n}$ converges to $y$, for all $t>0$. Then

$$
M_{2}\left(f\left(z_{n}\right), f(x), t\right)>M_{2}(f(y), f(x), t)
$$

and so $f\left(z_{n}\right) \in B\left(f(x), 1-M_{2}(f(x), f(y), t), t\right)$ for all $t>0$. Since $f$ is continuous, $\left(f\left(z_{n}\right)\right)_{n}$ converges to $f(y)$, and so $f(y) \in \overline{B\left(f(x), 1-M_{2}(f(x), f(y), t), t\right)}$ for all $t>0$. Therefore $\left(X_{2}, M_{2}, *\right)$ is a round fuzzy metric space.

Definition 3.8: Let $X$ be a nonempty set and $M_{1}$ and $M_{2}$ be two fuzzy metrics on $X$. Then $M_{1}$ and $M_{2}$ are said to be equivalent if there exist $a, b \in$ $(0,1]$ such that

$$
a \cdot M_{2}(x, y, t) \leq M_{1}(x, y, t) \leq b \cdot M_{2}(x, y, t)
$$

for all $x, y \in X$ and $t>0$. We denote it by $M_{1} \sim M_{2}$.

Corollary 3.9: The relation " $\sim$ " is defined as above is an equivalence relation.

Proof. The proof is the same as in classic metric spaces.

Corollary 3.10: Let $M_{1}$ be a round fuzzy metric for $X$. If $M_{2}$ is a fuzzy metric on $X$ equivalent to $M_{1}$ such that $M_{2}(x, z, t)>M_{2}(x, y, t)$ whenever $M_{1}(x, z, t)>M_{1}(x, y, t)$, then $M_{2}$ is also a round fuzzy metric for $X$.

Proof. Let $f:\left(X, M_{1}, *\right) \rightarrow\left(X, M_{2}, *\right)$ be the identity map. Then the proof is clear from Theorem 3.7.

Problem: How can be characterize the roundness in fuzzy metric spaces?

\section{REFERENCES}

[1] M. A. Erceg, Metric spaces in fuzzy set theory, J. Math. Anal. Appl., 69 (1979), $205-$ 230.

[2] A. George and P. Veeramani, On some results in fuzzy metric spaces, Fuzzy Sets and Systems, 64 (1994), $395-399$.

[3] A. George and P. Veeramani, On some results of analysis for fuzzy metric spaces, Fuzzy Sets and Systems, 90 (1997), $365-368$. 
[4] M. Grabiec, Fixed points in fuzzy metric spaces, Fuzzy Sets and Systems, 27 (1988), $385-389$.

[5] V. Gregori and S. Romaguera, Some Properties of fuzzy metric spaces, Fuzzy Sets and Systems, 115 (2000), $485-489$.

[6] O. Kramosil and J. Michalek, Fuzzy metric and statistical metric spaces, Kybernetica, 11 (1975), $326-334$.

[7] M.B. Nathanson, Round metric spaces, The American Mathematical Monthly, 82 (1975), $738-741$.

[8] B. Schweizer and A. Sklar, Statistical metric spaces, Pacific J. Math., 10(1960), $314-$ 334.

[9] P. Veeramani, Best approximation in fuzzy metric spaces, J. Fuzzy Math., 9 (2001), $75-80$.

[10] L. A. Zadeh, Fuzzy sets, Inform and Control, 8 (1965), 338 - 353.

\section{Received: October 21, 2006}

